\title{
Mutually Unbiased Coarse-Grained Measurements of Two or More Phase-Space Variables
}

\author{
E. C. Paul, ${ }^{1,}$ ' S. P. Walborn, ${ }^{1}$ D. S. Tasca, ${ }^{2}$ and Eukasz Rudnicki ${ }^{3,4, \dagger}$ \\ ${ }^{1}$ Instituto de Física, Universidade Federal do Rio de Janeiro, \\ Caixa Postal 68528, Rio de Janeiro, RJ 21941-972, Brazil \\ ${ }^{2}$ Instituto de Física, Universidade Federal Fluminense, Niteroi, RJ 24210-346, Brazil \\ ${ }^{3}$ Max-Planck-Institut für die Physik des Lichts, Staudtstraße 2, 91058 Erlangen, Germany \\ ${ }^{4}$ Center for Theoretical Physics, Polish Academy of Sciences, Aleja Lotników 32/46, 02-668 Warsaw, Poland
}

\begin{abstract}
Mutual unbiasedness of the eigenstates of phase-space operators-such as position and momentum, or their standard coarse grained versions-exists only in the limiting case of infinite squeezing. In [Phys. Rev. Lett. 120, 040403 (2018)] it was shown that mutual unbiasedness can be recovered for periodic coarse grainings of these two operators. Here we investigate mutual unbiasedness of coarsegrained measurements for more than two phase-space variables. We show that mutual unbiasedness can be recovered between periodic coarse graining of any two non-parallel phase-space operators. We illustrate these results through optics experiments, using the fractional Fourier transform to prepare and measure mutually unbiased phase-space variables. The differences between two and three mutually unbiased measurements is discussed. Our results contribute to bridging the gap between continuous and discrete quantum mechanics and could be useful in quantum information protocols.
\end{abstract}

\section{INTRODUCTION}

Complementarity-the fact that perfect knowledge about a certain observable prohibits the knowledge of a second complementary observable-is a cornerstone of Quantum Mechanics and Quantum Information. Complementarity can be better formulated through the concept of mutual unbiasedness [1, which can be characterized in terms of bases (projectors) or more generalized measurements [2]. Mutually unbiased bases (MUBs) [3] play an important role in the security of quantum cryptography [4, 5], the efficiency of quantum tomography [6, 7], and are useful for identifying quantum correlations such as entanglement [8-10] and steering [11-18], as well as for certifying quantum randomness [19].

In a finite $d$-dimensional Hilbert space, two orthonormal bases $\left\{\left|a_{i}\right\rangle\right\}$ and $\left\{\left|b_{j}\right\rangle\right\}$ are mutually unbiased if and only if $\left|\left\langle a_{i} \mid b_{j}\right\rangle\right|=1 / \sqrt{d}$, for $i, j=0, \ldots, d-1[20$. For the case of continuous variables $(\mathrm{CV})$, it is well known that the conjugate pair of position and momentum eigenstates also presents mutual unbiasedness, as per the relation $|\langle x \mid p\rangle|=1 / \sqrt{2 \pi}$, where here and throughout we set $\hbar=1$. The kets $|x\rangle$ and $|p\rangle$ are normalized (to Dirac delta) eigenvectors of $\hat{x}$ (position) and $\hat{p}$ (momentum) operators, respectively. In fact, if we define rotated phasespace operators as linear combinations of dimensionless position and momentum operators,

$$
\hat{q}_{\theta}=\cos \theta \hat{x}+\sin \theta \hat{p}
$$

the eigenbases of $\hat{q}_{\theta}$ and $\hat{q}_{\theta^{\prime}}$ are always mutually unbiased, provided that $\sin \left(\theta-\theta^{\prime}\right) \neq 0$.

\footnotetext{
*Electronic address: eduardo.paul@if.ufrj.br

${ }^{\dagger}$ Electronic address: rudnicki@cft.edu.pl
}

While mutual unbiasedness is not limited to only two phase-space operators in the CV case, there is a large qualitative difference when compared to discrete, finitedimensional quantum mechanics. In particular, for a $d$ dimensional system, the condition for MUBs can be mutually satisfied by $d+1$ bases whenever $d$ is the power of a prime number 20]. For CVs, even though one might suspect that the infinite-dimensional nature of the state space would lead to infinitely many MUBs, this is not the case. Indeed, Weigert and Wilkinson [21] have shown that one can identify at most three mutually unbiased bases for each CV system. This result applies to the bases mutually related by rotations on the phase space, leading to a mutually unbiased phase-space "triple" set of operators. For example, in addition to the eigenbasis of the position operator $\hat{x} \equiv \hat{q}_{0}$, one can consider eigenbases of two more operators: $\hat{r} \equiv \hat{q}_{2 \pi / 3}$ and $\hat{s} \equiv \hat{q}_{4 \pi / 3}$, as illustrated in Fig. 1. The choice to begin with the position operator has been made without loss of generality - alternatively one could start with any other $\hat{q}_{\theta}$, and rotate $\hat{r}$ and $\hat{s}$ by $\theta$. Furthermore, if squeezing operations are allowed, mutual unbiasedness can be preserved even for phase space triples whose angles do not differ by $2 \pi / 3$ [21.

The above discussion on MUBs in the context of CVs was based on the definition of eigenstates of phasespace operators. These states are normalized to Dirac delta distributions and as such are not accessible in an experiment, as they correspond to the regime of infinite squeezing [22]. When considering the physical case of quantum states that are merely "localized" around some-say-position, the corresponding MUB condition with the momentum is lost. This is true for all mutually unbiased pairs as well as for triples.

A related issue arises in experimental scenarios where it is beneficial or even necessary to use coarse-grained measurements [13, 14, 23, 25]. In [25], while working 


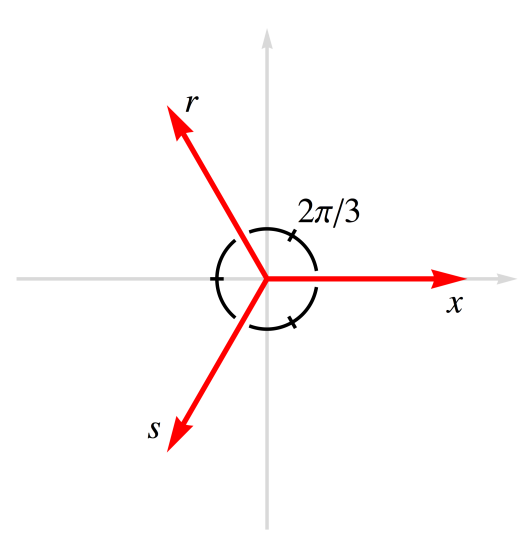

Figure 1: Pictorical representation of the phase-space variables defining the 3 mutually unbiased bases in continuous variables. The arrows divide the phase space in three equal slices of $2 \pi / 3$.

with the pair $(x, p)$ of canonically conjugate variables, it was shown that "standard" coarse graining does not preserve the unbiasedness property originally present in CVs. Instead, one can work with coarse-grained periodic structures and recover unbiasedness in that regime, in some way opposite to that known from modular variables [26 33. The goal of the present paper is to expand the theoretical and experimental analysis performed in Ref. [25] to the case of general phase-space variables, and in particular, to coarse-grained measurements of more than two phase-space operators.

This paper is organized in the following way. In section II we introduce periodic coarse-grained measurements along arbitrary directions in phase space. In section III we define mutual unbiasedness of pairs of coarse-grained operators, and extend this definition to more than two measurements in section IV. In section V we present experiments and results investigating both the mutual unbiasedness of PCG phase-space triples, and the mutual unbiasedness of PCG measurements corresponding to phase-space directions related by an arbitrary angle. Finally, we provide concluding remarks in section VI.

\section{PERIODIC COARSE-GRAINING OF PHASE-SPACE VARIABLES}

Let $\left|q_{\theta}\right\rangle$ denote an eigenstate of the quadrature operator $\hat{q}_{\theta}$ defined in Eq. (1). The scalar product between eigenstates corresponding to different quadrature operators is $\left\langle q_{\theta^{\prime}} \mid q_{\theta}\right\rangle=\mathcal{F}\left(q_{\theta^{\prime}}, q_{\theta}\right)$, where

$$
\mathcal{F}\left(q_{\theta^{\prime}}, q_{\theta}\right)=\sqrt{\frac{i e^{i \Delta \theta}}{2 \pi|\sin \Delta \theta|}} e^{i \frac{\cot \Delta \theta}{2}\left(q_{\theta}^{2}+q_{\theta^{\prime}}^{2}\right)-i \frac{q_{\theta} q_{\theta^{\prime}}}{\sin \Delta \theta}},
$$

is the kernel of the fractional Fourier transform [34, and $\Delta \theta=\theta^{\prime}-\theta$. As $\left|\left\langle q_{\theta^{\prime}} \mid q_{\theta}\right\rangle\right|=1 / \sqrt{2 \pi|\sin \Delta \theta|}$, it is clear

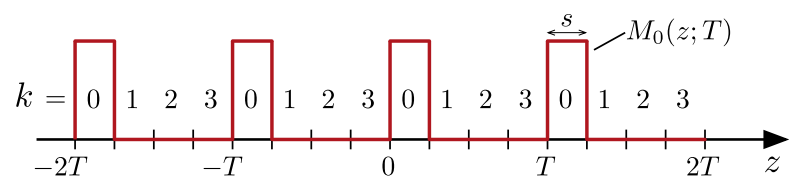

Figure 2: Mask function $M_{0}(z ; T)$, as defined in Eq. 4, for the $d=4$ case. Here, $T$ is the mask's spatial period, $s$ is its bin width, $z$ is the spatial coordinate along which the mask is defined, and $k$ is the index that identifies individual masks. This mask can be viewed as a periodic array of apertures spaced by $d=4$ bins of size $s$.

that the eigenbases of any two operators $\hat{q}_{\theta}$ and $\hat{q}_{\theta^{\prime}}$ are mutually unbiased whenever $\sin \Delta \theta \neq 0$.

We can use the eigenstates of $\hat{q}_{\theta}$ to define a family (labeled by $\theta$ ) of $d$ coarse-grained projective measurement operators

$$
\hat{\Omega}_{k}^{\theta}=\int d q_{\theta} M_{k}\left(q_{\theta}-q_{\theta}^{\mathrm{cen}} ; T_{\theta}\right)\left|q_{\theta}\right\rangle\left\langle q_{\theta}\right|,
$$

with detector apertures encoded in "mask functions" $M_{k}$, such that $\sum_{k=0}^{d-1} M_{k}=1$. The displacement parameter $q_{\theta}^{\text {cen }}$ is included to represent the freedom of setting the origin, and the parameter $T_{\theta}$ is the period of the mask function. One possible choice for the mask functions is

$$
M_{k}(z ; T)= \begin{cases}1, & k s \leq z(\bmod T) \leq(k+1) s \\ 0, & \text { otherwise }\end{cases}
$$

As defined in Ref. 25, the functions (4) are periodic square waves specified by the period $T$ and bin width $s=T / d$, so that $d$ can be considered as a "dimensionality" parameter. The outcome probabilities produced by the set of projectors 3 with the periodic mask functions (4) define the PCG of the probability distribution associated with the phase-space variable $q_{\theta}$. Since we work with dimensionless variables, both mask parameters $T$ and $s$ are also dimensionless. In Fig. 2 we illustrate the PCG geometry and the periodic mask function for the particular case of $d=4$.

The operators $\hat{\Omega}_{k}^{\theta}$ are diagonal in their associated basis given by $\left\{\left|q_{\theta}\right\rangle\right\}$. In order to discuss mutual unbiasedness between several PCG measurements corresponding to different directions in phase space, it is beneficial to express $\hat{\Omega}_{k}^{\theta}$ in terms of an arbitrarily rotated basis $\left|q_{\theta^{\prime}}\right\rangle$. To this end, one needs to represent the periodic mask function by means of its Fourier series decomposition

$$
M_{k}(z ; T)=\sum_{N \in \mathbb{Z}} f_{N} e^{-\frac{2 \pi i N}{d} k} e^{\frac{2 \pi i N}{T} z}
$$

with

$$
f_{N}=\frac{1-e^{-\frac{2 \pi i N}{d}}}{2 \pi i N} .
$$

A moderately straightforward but lengthy calculation (see Appendix A leads to the result 


$$
\hat{\Omega}_{k}^{\theta}=\sum_{N \in \mathbb{Z}} f_{N} \int d q_{\theta^{\prime}} e^{i N \phi_{k}^{(N)}\left(q_{\theta^{\prime}}\right)}\left|q_{\theta^{\prime}}\right\rangle\left\langle q_{\theta^{\prime}}-N \tau_{\theta}\right|,
$$

with

$\phi_{k}^{(N)}\left(q_{\theta^{\prime}}\right)=\tau_{\theta}\left(q_{\theta^{\prime}}-\frac{N \tau_{\theta}}{2}\right) \cot \Delta \theta-\left(2 \pi k / d+\frac{q_{\theta}^{\mathrm{cen}} \tau_{\theta}}{\sin \Delta \theta}\right)$,

and $\tau_{\theta}=2 \pi \sin \Delta \theta / T_{\theta}$. Note that in the limit $\theta^{\prime} \rightarrow \theta$, or equivalently $\Delta \theta \rightarrow 0$, the above expressions are not singular, since $\tau_{\theta} \rightarrow 0$ in this limit.

\section{MUTUAL UNBIASEDNESS}

Given a pure state $|\Psi\rangle$, we define a family of probabilities

$$
p_{k}^{(\theta)}(\Psi)=\left\langle\Psi\left|\hat{\Omega}_{k}^{\theta}\right| \Psi\right\rangle
$$

which encodes the results of the PCG measurements in question. Following [25], measurements labeled by different angles in phase space, $\theta$ and $\theta^{\prime}$, are mutually unbiased if for all $|\Psi\rangle$ and all $k_{0}, l_{0}=0, \ldots, d-1$,

$$
\begin{aligned}
& p_{k}^{\left(\theta^{\prime}\right)}(\Psi)=\delta_{k_{0} k} \Longrightarrow p_{l}^{(\theta)}(\Psi)=d^{-1} \\
& p_{l}^{(\theta)}(\Psi)=\delta_{l_{0} l} \Longrightarrow p_{k}^{\left(\theta^{\prime}\right)}(\Psi)=d^{-1}
\end{aligned}
$$

In words, whenever a state is localized according to the measurements with respect to the phase-space variable specified by the angle $\theta^{\prime}$, it is evenly spread with respect to measurements corresponding to the second variable defined by $\theta$. Note that whenever the pairs of projective measurements are unitarily equivalent, it is sufficient to consider a single condition, say $8 \mathrm{a}$. In phase space this is usually the case since $\hat{q}_{\theta^{\prime}}=\hat{F}_{\Delta \theta}^{\dagger} \hat{q}_{\theta} \hat{F}_{\Delta \theta}$, with $\hat{F}_{\Delta \theta}$ being the unitary fractional Fourier transform operator. However, for the particular projective measurements considered here, one also needs an extra symmetry. Namely, the presumed condition on the periods $T_{\theta}$ and $T_{\theta^{\prime}}$, which is necessary to fulfill $8 \mathrm{a}$, must be invariant under the swap of both periods 25]. The last requirement will be verified below. Here we mainly emphasize that in the discussed scenario the requirement $8 \mathrm{~b}$ follows automatically, provided that $8 \mathrm{a}$ ) is satisfied.

We are in position to establish the main theoretical results of this paper. Due to Eq. (6) we can write

$p_{l}^{(\theta)}(\Psi)=\sum_{N \in \mathbb{Z}} f_{N} \int d q_{\theta^{\prime}} e^{i N \phi_{l}^{(N)}\left(q_{\theta^{\prime}}\right)} \psi^{*}\left(q_{\theta^{\prime}}\right) \psi\left(q_{\theta^{\prime}}-N \tau_{\theta}\right)$,

with $\psi^{*}\left(q_{\theta^{\prime}}\right)=\left\langle q_{\theta^{\prime}} \mid \Psi\right\rangle$. This expression is a direct extension of the formula derived in 25 for the special case of the conjugate pair of position and momentum. Indeed, if $\theta^{\prime}=0$ and $\theta=\pi / 2$, so that $q_{\theta^{\prime}} \equiv x$, we find that $\tau_{\theta}$ is negative and equal to $-2 \pi / T_{p}$, with $T_{p} \equiv T_{\pi / 2}$.

Returning to the general case, we immediately conclude from Eq. (9) that if

$\frac{T_{\theta^{\prime}} T_{\theta}}{2 \pi}=\frac{d|\sin \Delta \theta|}{m}, \quad m \in \mathbb{N}, \quad$ s.t. $\quad \forall_{n=1, \ldots, d-1} \frac{m n}{d} \notin \mathbb{N}$,

then the mutual-unbiasedness condition (8) is fulfilled. In words, $m$ is a natural number [38] such that $m n / d \notin \mathbb{N}$ for all $n=1, \ldots, d-1$. As explained in [25], there is no clear pattern followed by the allowed values of $m$. However, the case $m=1$ stands out as it is present for all values of $d$. An excluded case in which $m$ is a multiple of $d$ shall correspond to pairs of modular variables on the phase space, as this happens for the standard scenario $\theta^{\prime}=0$ and $\theta=\pi / 2$ [25, 27]. Again, both displacements of the origins are absent in 10 .

To prove the above statement we observe that under the condition in question, an autocorrelation term present in (9) simplifies to $\psi^{*}\left(q_{\theta^{\prime}}\right) \psi\left(q_{\theta^{\prime}}-m N \epsilon_{\Delta \theta} T_{\theta^{\prime}} / d\right)$ with $\epsilon_{\Delta \theta}=\operatorname{sign}(\sin \Delta \theta)$. Now, if $p_{k}^{\left(\theta^{\prime}\right)}(\Psi)=\delta_{k_{0} k}$ for some $k_{0}$, then the autocorrelation differs from zero only for integer values of $m N / d$. The sign $\epsilon_{\Delta \theta}$ plays no role here. Due to the additional requirement put on $m$, the quantity $m N / d$ is an integer only when $N / d \in \mathbb{Z}$. In turn, the prefactor $f_{N}$ defined in (5b) vanishes for all $N$ that are multiples of $d$, except the case $N=0$, when it assumes the value of $1 / d$. This proves the desired result, since for $N=0$, the $q_{\theta^{\prime}}$ integral in $\sqrt{9}$ is equal to 1 . As already mentioned, the condition 10 is invariant with respect to the swap of both periods, as it only depends on their product.

\section{MUTUAL UNBIASEDNESS FOR SEVERAL VARIABLES}

For infinite-dimensional CV systems there are up to three simultaneously mutually unbiased bases [21], given-for example-as eigenbases of the operators $\hat{x} \equiv \hat{q}_{0}$, $\hat{r} \equiv \hat{q}_{2 \pi / 3}$ and $\hat{s} \equiv \hat{q}_{4 \pi / 3}$. Contrarily, in finite-dimensional quantum mechanics one can find even more MUBs [20]. The coarse-grained scenario is the discretization of the CV case, situated somewhat between these two distinct regimes, and thus it is especially interesting to discover which pattern of mutual unbiasedness will be reproduced. We start with the general case of the periodic coarsegrained version of mutually unbiased CV variables, while later focus on the particular case of $m=1$ [see Eq. (10)], valid for all $d$, and prove that, similarly to the usual CVs, only three PCG measurements can be simultaneously mutually unbiased. In the most general scenario - those with independent values of $m$ for each pair of variables - there is room for more complex settings (possibly more than three unbiased measurements). We will not explore this plethora of possibilities here. 


\section{A. Three mutually unbiased CV}

Let $T_{x}, T_{r}$ and $T_{s}$ be the periods corresponding to the mask functions of PCG measurements along $x, r$ and $s$. Since for each pair of the variables $|\sin \Delta \theta|=\sqrt{3} / 2$, equations 10 for $\theta, \theta^{\prime} \in\{0,2 \pi / 3,4 \pi / 3\}$ become

$$
T_{x} T_{r}=\frac{\sqrt{3} \pi d}{m_{1}}, \quad T_{x} T_{s}=\frac{\sqrt{3} \pi d}{m_{2}}, \quad T_{r} T_{s}=\frac{\sqrt{3} \pi d}{m_{3}},
$$

where $m_{1}, m_{2}, m_{3}$ are natural numbers satisfying the relevant constraints in Eq. 10 . The general solution is found to be:

$$
T_{x}=\sqrt{\sqrt{3} \pi d \frac{m_{3}}{m_{1} m_{2}}}, \quad T_{r}=\frac{m_{2}}{m_{3}} T_{x}, \quad T_{s}=\frac{m_{1}}{m_{3}} T_{x} .
$$

Clearly, for the special case $m_{1}=m_{2}=m_{3}=1$, all the periods assume the fixed, dimension-dependent value of $\sqrt{\sqrt{3} \pi d}$. Contrary to the case of the mutually unbiased pair, in which one of the periods is not fixed but serves as a reference length [25], for the mutually unbiased triple all the periods are fixed, up to the freedom offered by the natural numbers $m$. This situation is very similar to saturation of variance-based uncertainty relations. For two variables, all pure Gaussian states saturate a version of the Heisenberg uncertainty relation, regardless of the variance of the state. For three $\mathrm{CV}$ variables, the product of three variances is bounded by $1 / 8,9,21,35]$, and the inequality $(\Delta x)^{2}(\Delta r)^{2}(\Delta s)^{2} \geq 1 / 8$ is saturated only when all the involved variances are equal to $1 / 2$.

\section{B. Maximum Number of MUBs in the case $m=1$}

Here we show that for the periodic coarse graining considered and with $m=1$, a maximum number of three MUBs is possible. We proceed by searching for an arbitrary number of $K$ coarse-grained, periodic MUBs, specified by the angles $\theta_{1}, \ldots, \theta_{K}$ and the periods $T_{1}, \ldots, T_{K}$. Without loss of generality we assume that $\theta_{j}>\theta_{i}$ whenever $j>i$, and that $0 \leq \theta_{i}<2 \pi$ for all $i=1, \ldots, K$.

We would like to check what number $K$ of PCG measurements $\left\{\hat{\Omega}_{k}^{\theta_{j}}\right\}$ can simultaneously be mutually unbiased with the same value $m=1$, i.e. the conditions

$$
\frac{T_{i} T_{j}}{2 \pi}=d\left|\sin \left(\theta_{i}-\theta_{j}\right)\right|
$$

are all satisfied. Using $i=1$ we get $(j \geq 2)$ :

$$
\frac{T_{j}}{2 \pi}=\frac{d}{T_{1}}\left|\sin \left(\theta_{1}-\theta_{j}\right)\right|
$$

so that, after minor trigonometric simplification, we are left with $(K-1)(K-2) / 2$ consistency conditions $(j>$ $i \geq 2)$ :

$$
\left|\cot \left(\theta_{j}-\theta_{1}\right)-\cot \left(\theta_{i}-\theta_{1}\right)\right|=\frac{2 \pi d}{T_{1}^{2}}
$$

It is clear that if $K=3$ we have a single condition for $i=2$ and $j=3$, which in turn can trivially be solved for $T_{1}$. In that way one immediately obtains a generalized $(\theta$-dependent) variant of the solutions 12 .

Analyzing the case $K=4$, it is convenient to make a simplifying assumption which does not spoil the generality of the argument: let us assume that $\theta_{1}=0$, i.e. our first member is the periodic coarse graining of the position variable. In this case we are left with three consistency conditions, which, after solving one of them with respect to $T_{1}$, lead to two equations:

$$
\begin{aligned}
& \left|\cot \theta_{3}-\cot \theta_{2}\right|=\left|\cot \theta_{4}-\cot \theta_{2}\right|, \\
& \left|\cot \theta_{4}-\cot \theta_{3}\right|=\left|\cot \theta_{4}-\cot \theta_{2}\right| .
\end{aligned}
$$

All three angles $\theta_{2}, \theta_{3}, \theta_{4}$ can neither be 0 nor $\pi$ as in such a case some of the directions would reproduce the position variable $($ or $-x)$. As a result, no infinities occur in the above conditions. By a similar argument we exclude $\cot \theta_{2}=0$, since it implies $\cot \theta_{3}=0=\cot \theta_{4}$, and all three directions would need to correspond to $\pm p$. Since $\cot \theta_{2} \neq 0$ we can introduce auxiliary variables $\zeta_{3}=\cot \theta_{3} / \cot \theta_{2}$ and $\zeta_{4}=\cot \theta_{4} / \cot \theta_{2}$, and rewrite the consistency conditions in the form

$$
\left|\zeta_{3}-1\right|=\left|\zeta_{4}-1\right|=\left|\zeta_{4}-\zeta_{3}\right|
$$

It is easy to verify that the only solution to these conditions is given by $\zeta_{3}=1=\zeta_{4}$. As a result we need to find three angles from the range $] 0,2 \pi$ [ for which their cotangent would assume the same value. Since in the desired range the cotangent function assumes every real, finite value exactly twice, it is impossible to fit four coarsegrained MUBs (with $m=1$ ) into the phase space. This argument can be applied to any number $K>3$ directions in phase space. Thus, for the PCG considered, there are at most 3 mutually unbiased measurements.

\section{EXPERIMENT}

\section{A. Three mutually unbiased PCG measurements}

To demonstrate mutually unbiased PCG measurements of the phase-space triple $x(\theta=0), r(\theta=2 \pi / 3)$ and $s(\theta=4 \pi / 3)$, we performed an optics experiment exploring the transverse spatial variables of a paraxial laser beam. Using systems of converging lenses and spatial light modulators (SLMs), we prepared the eigenstate of the PCG measurement operator $\hat{\Omega}_{k}^{\theta}$, described by Eq. (3) with Eq. (4). In the sequence, we used an equivalent apparatus to perform the measurement described by another operator $\hat{\Omega}_{k^{\prime}}^{\theta^{\prime}}$ on the prepared state. This procedure was followed for every combination of (different) $\theta, \theta^{\prime} \in\{0,2 \pi / 3,4 \pi / 3\}$, and every possible value of $k$ and $k^{\prime}$ for measurement dimensionality $d$ from 2 up to 10 . The 


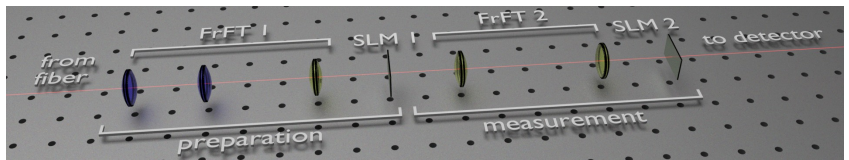

Figure 3: Sketch of the experimental setup. The actual spatial light modulators (SLM) used were reflective. The fractional Fourier Transforms (FrFT) are described in the text.

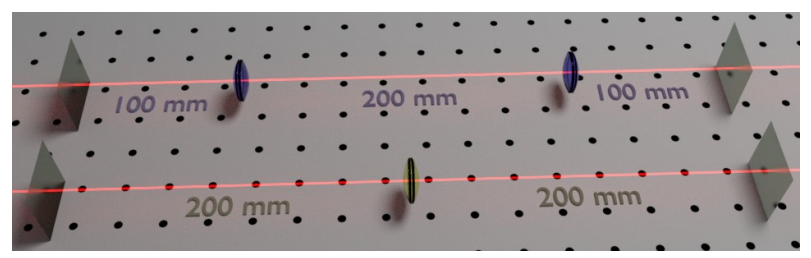

Figure 4: Lens systems used to perform the FrFTs. On the top, two confocal 100-mm focal-length lenses are used to perform a $\pi$ rotation. On the bottom, a single $400-\mathrm{mm}$ lens is used to perform a $\pi / 3$ rotation. The planes represent the object and image planes of the systems. Sequential combinations of these systems were used to produce the desired phase-space rotations.

PCG measurements $\left\{\hat{\Omega}_{k}^{\theta}\right\}$ and $\left\{\hat{\Omega}_{k^{\prime}}^{\theta^{\prime}}\right\}$ can be considered unbiased if the resulting conditional probability distributions satisfy $p\left(k^{\prime} \mid k\right)=1 / d \forall k, k^{\prime}$.

Fig. 3 shows a simplified scheme of our experimental setup. The output of an attenuated Thorlabs' CPS180 635-nm diode laser was first coupled in and out of a single-mode fiber. At the output of the fiber the beam was well-collimated so that its transverse field distribution was given by $\exp \left[-x^{2} /\left(4 \sigma^{2}\right)\right]$, where $\sigma=875 \mu \mathrm{m}$. Though our experiment used a laser beam, the same physics applies to single photons with equivalent spatial and spectral properties.

The preparation of the eigenstate of operator $\hat{\Omega}_{k}^{\theta}$ was done in two steps. First, the beam was subjected to a fractional Fourier transform (FrFT) by a set of converging lenses connecting a reference plane to the plane of SLM 1. In this way, the chosen phase-space direction, defined by $\theta$, was mapped onto the phase-space $x$ axis on the physical position of SLM 1, which then performed the operation described by $\hat{\Omega}_{k}^{0}$. This procedure allows us to prepare eigenstates of the operator $\hat{\Omega}_{k}^{\theta}$.

A single lens of focal length $f$, placed symmetrically at a distance $z$ from the input and output planes, performs a FrFT characterized by the phase-space rotation angle $\theta$ (with proper dimensionalization discussed below), where [36]

$$
z=2 f \sin \left(\theta^{2} / 2\right) .
$$

In order to achieve all of the phase-space rotations desired, we combined two different systems of lenses. On one hand, a pair of confocal 100-mm focal-length lenses together performed a $\pi$ rotation (see Fig. (4). On the other

\begin{tabular}{|c|c|c|c|}
\hline$d$ & $T(\mu \mathrm{m})$ & $T / l$ & $T_{\exp }(\mu \mathrm{m})$ \\
\hline \hline 2 & 617.3 & 77.2 & 616 \\
\hline 3 & 756.0 & 94.5 & 752 \\
\hline 4 & 872.9 & 109.1 & 872 \\
\hline 5 & 976.0 & 122.0 & 976 \\
\hline 6 & 1069.1 & 133.6 & 1072 \\
\hline 7 & 1154.8 & 144.3 & 1152 \\
\hline 8 & 1234.5 & 154.3 & 1232 \\
\hline 9 & 1309.4 & 163.7 & 1312 \\
\hline 10 & 1380.2 & 172.5 & 1384 \\
\hline
\end{tabular}

Table I: Ideal mask periods $(T)$, as calculated from the theory, and their ratio to the SLM pixel length $(T / l)$, as a function of the dimension $(d)$. The actual experimental periods used $\left(T_{\text {exp }}\right)$ correspond to the nearest possible integer number of pixels.

hand, a single 400-mm focal-length lens, placed $200 \mathrm{~mm}$ away from both its input and output planes performed a $\pi / 3$ rotation [37] (see also Fig. 4). Therefore, two sequential $400-\mathrm{mm}$ lenses performed a $2 \pi / 3$ rotation, a pair of 100-mm lenses followed by a 400-mm lens performed a $4 \pi / 3$ rotation, and two sequential pairs of $100-\mathrm{mm}$ lenses were used to perform a $2 \pi$ rotation.

To adequately interpret the action of a single lens as a FrFT and therefore as a rotation in phase space, it is necessary to use dimensionless variables. This can be done by using the scaling factor 37]

$$
\delta=\sqrt{\lambda f \sin \theta / 2 \pi},
$$

where $\lambda$ is the laser beam's wavelength, $f$ is the lens' focal length and $\theta$ is the corresponding phase-space rotation angle. Choosing $f=400 \mathrm{~mm}$ and $\theta=\pi / 3$ we were able to use the same scaling factor for all variables. This means the dimensionless variables are $x=x^{\prime} / \delta, r=r^{\prime} / \delta$ and $s=s^{\prime} / \delta$, where the primed letters represent the measured transverse spatial variables (with dimension of length).

To perform the operations described by $\hat{\Omega}_{k}^{0}$, we used reflective Holoeye Pluto phase-only SLMs, which generated the desired mask functions aligned with the horizontal (lab table). All the mask periods were given by Eq. (12), with $m_{1}=m_{2}=m_{3}=1$. Combining equations Eqs. (12) and (19), we can calculate the periods for each dimension. Considering that we were constrained to using periods that were integer multiples of the SLM pixel length $(l=8 \mu \mathrm{m})$, the actual periods used were approximations of the theoretical ones, as we can see in Table I.

After the prepare and measure procedures, the beam size was then reduced by a factor of $\approx 10$ (a magnification of $\approx 0.1$ ) by an imaging system consisting of two confocal lenses of focal lengths $250 \mathrm{~mm}$ and $25.4 \mathrm{~mm}$, and coupled into a $300-\mu \mathrm{m}$ core multi-mode fiber using a 10X Olympus plane achromat objective. The multi-mode fiber was 


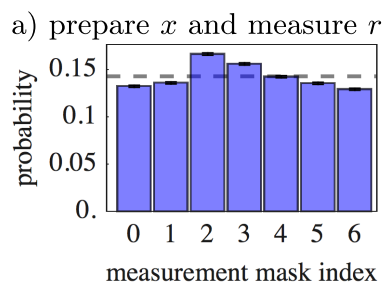

b) prepare $r$ and measure $x$

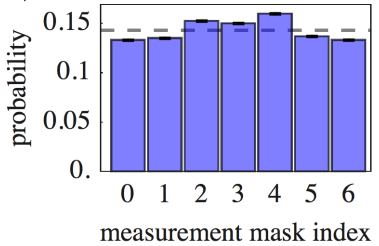

c) prepare $x$ and measure $s$

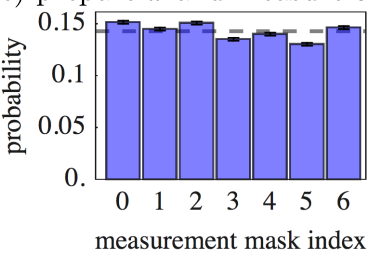

e) prepare $r$ and measure $s$

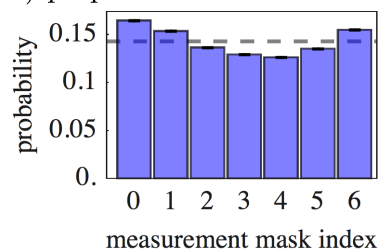

d) prepare $s$ and measure $x$

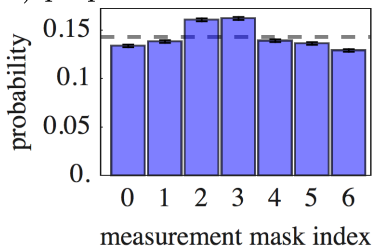

f) prepare $s$ and measure $r$

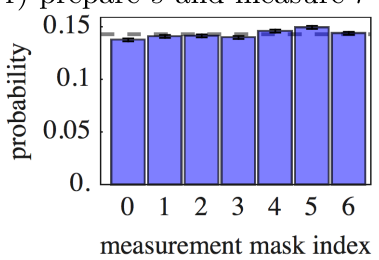

Figure 5: Probability distributions obtained for a periodic coarse graining with dimension $d=7$, when using preparation mask $k=2$. Each of the graphs corresponds to one of the possible combinations of the prepare and measure phasespace directions $x(\theta=0), r(\theta=2 \pi / 3)$ and $s(\theta=4 \pi / 3)$. The dashed lines correspond to the theoretical predictions. The error bars correspond to the standard deviations derived from the laser poissonian count statistics.

then connected to one channel of a Perkin Elmer SPCMAQ4C single-photon avalanche photodiode detector. The number of photons arriving at the detector in a $0.1 \mathrm{~s}$ time interval was registered.

For each preparation, all the measurement outcomes were normalized with respect to the sum of all outcomes (for the preparation at hand). The normalized values were then interpreted as the conditional probabilities $p\left(k^{\prime} \mid k\right)$ of obtaining each measurement result $k^{\prime}$ given the corresponding preparation $k$. Fig. 5 shows an example of the probability distributions obtained for $d=7$, where the prepared mask was $k=2$. One can see qualitatively that the measurement outcomes for all pairs of variables are approximately uniform, displaying three-fold mutual unbiasedness for these periodic coarse-grained measurements.

As a figure of merit to check the measurements' unbiasedness, we opted to use the Kullback-Leibler (KL) divergence, also known as the relative entropy:

$$
D(P \| Q)=\sum_{i=0}^{d-1} P_{i} \log \left(\frac{P_{i}}{Q_{i}}\right),
$$

where $P$ is the measured probability distribution, $Q$ is the target uniform distribution, such that $Q_{i}=1 / d$ for all $Q_{i}$, and we use the base-2 logarithm. A perfect

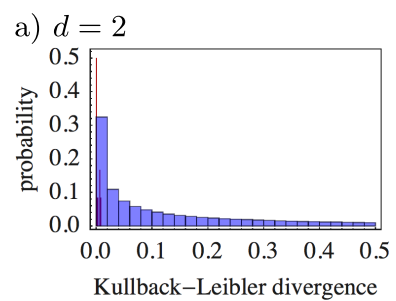

b) $d=3$
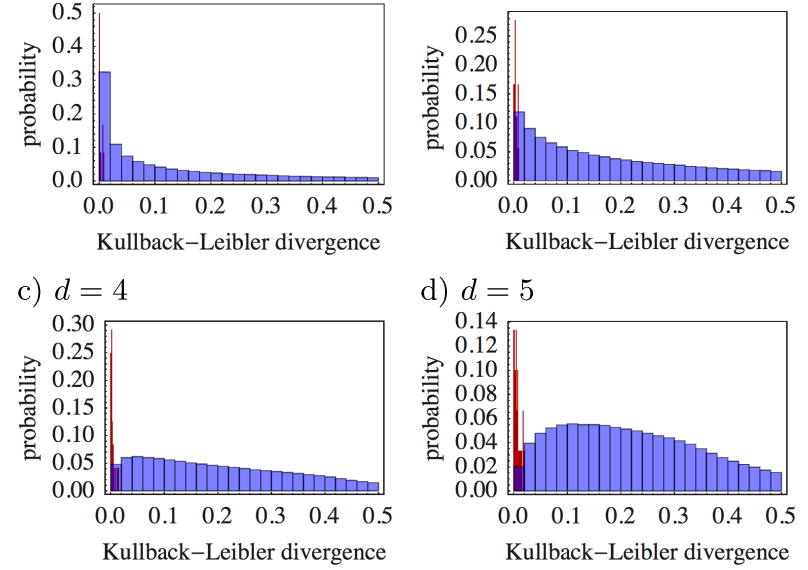

d) $d=5$
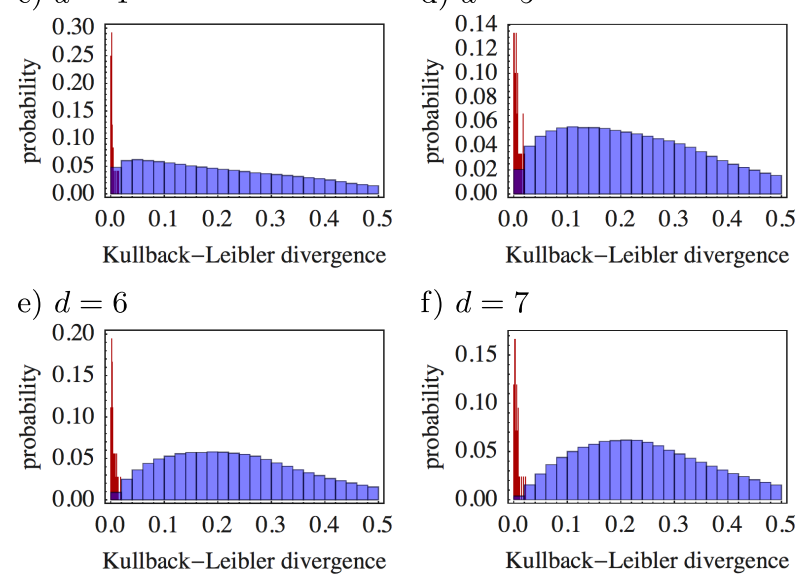

f) $d=7$
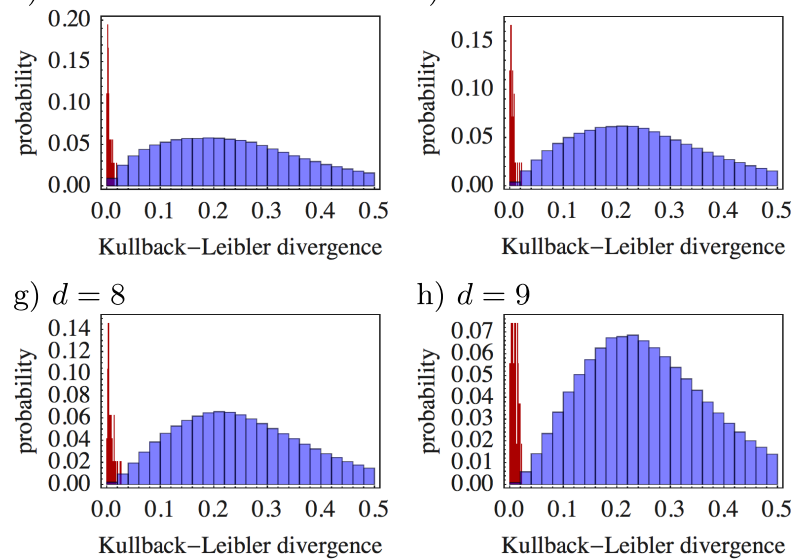

h) $d=9$
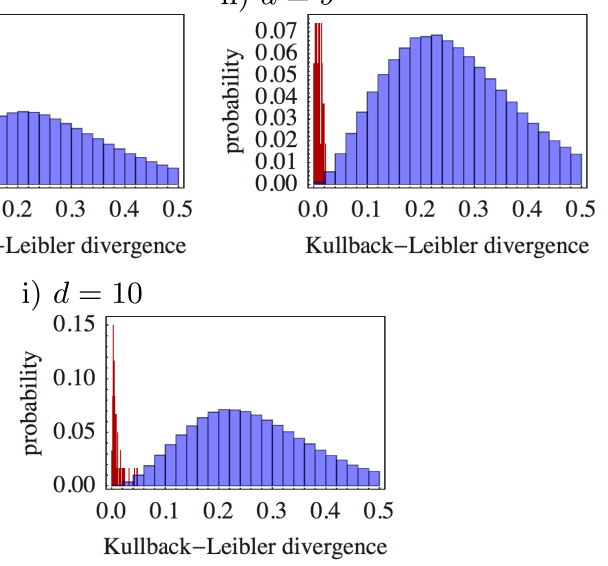

Figure 6: Histograms of the Kullback-Leibler divergences. The blue bars represent the $10^{6}$ simulated random distributions, while the red bars represent the experimental data. The horizontal axes are the KL divergences and the vertical axes are the probabilities of occurrence.

match between generated and target uniform distribution means zero divergence, while a maximal divergence of $D=\log (d)$ is achieved for a perfectly localized distribution. Using this quantity, we compared (for each dimension) the set of experimental probability distributions to a sample of $10^{6}$ simulated random probability distributions. Each of Figs. 6 (one for each value of d) shows the histogram of KL divergences for the sample of simulated probability distributions (blue bars), together with the histogram for the divergences calculated from the experimentally obtained probability distributions (red bars). We can clearly see from the graphs that the experimental probability distributions produce 


\begin{tabular}{|c|c|}
\hline$d$ & $P$ \\
\hline \hline 2 & $80 \pm 1$ \\
\hline 3 & $94.4 \pm 0.4$ \\
\hline 4 & $97.2 \pm 0.2$ \\
\hline 5 & $98.2 \pm 0.2$ \\
\hline 6 & $99.3 \pm 0.1$ \\
\hline 7 & $99.50 \pm 0.04$ \\
\hline 8 & $99.65 \pm 0.03$ \\
\hline 9 & $99.89 \pm 0.02$ \\
\hline 10 & $99.33 \pm 0.04$ \\
\hline
\end{tabular}

Table II: Percentage $(P)$ for each dimension $(d)$ of simulated probability distributions that produce KL divergences greater than the greatest value achieved for the experimental distributions. The errors listed correspond to the standard deviations.

KL divergences much lower than what would be expected from random chance.

To have a better quantitative view of these results, we calculated (again, for each $d$ ) the percentage of simulated probability distributions that produce KL divergences greater than the greatest value achieved for the experimental distributions (see Table II). If we allow the extrapolation of these results to the set of every (infinite) $d$-dimensional probability distribution, this means that each experimental distribution is closer to the uniform distribution, as per the KL divergence, than at least the given percentage of every possible $d$-dimensional probability distribution. These results show that we were able to obtain probability distributions very close to the uniform distribution, and that their proximity was not solely a matter of random chance, demonstrating that the three measurements defined are indeed mutually unbiased.

\section{B. Unbiasedness for two arbitrary directions in phase space}

Any two PCG measurements in distinct directions in phase space are mutually unbiased, given that Eq. (10) is satisfied. With that in mind, we carried out experiments in which we prepared eigenstates of $\hat{\Omega}_{k}^{0}$, for every $k=0, \ldots, d-1$, and then performed measurements described by the operators $\left\{\hat{\Omega}_{k^{\prime}}^{\alpha}\right\}$, with the smallest value for the angle $\alpha$ that we could achieve in our experimental setup. Using $z=200 \mathrm{~mm}$ and $f=250 \mathrm{~mm}$, Eq. (18) gives $\theta \approx 78.5^{\circ}$. Two such optical systems in a row implement a phase-space rotation of $157^{\circ}$. Following this operation by a reflection in real space, which is equivalent to the transformation $x \rightarrow-x$ and is automatically fulfilled due to the reflective character of the SLMs, we achieved the rotation angle of $\alpha \approx 23^{\circ}$. With this operation, we performed the same procedure described in the previous section, but repeating the measurements for several bin widths. Each one of Figs. 7 shows the KL
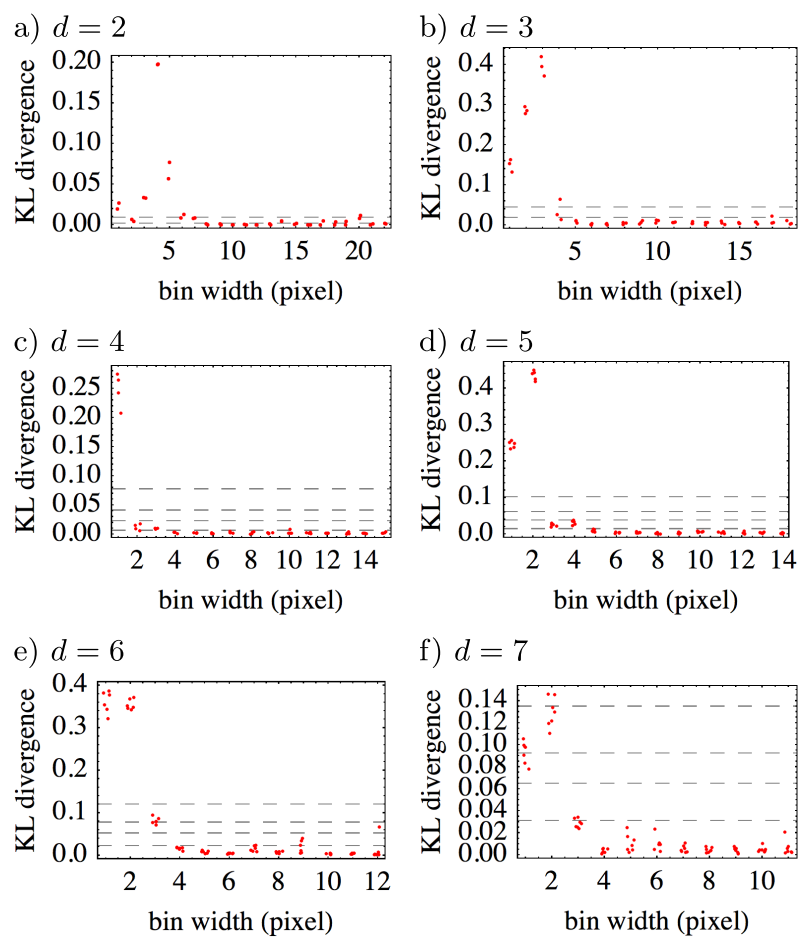

f) $d=7$
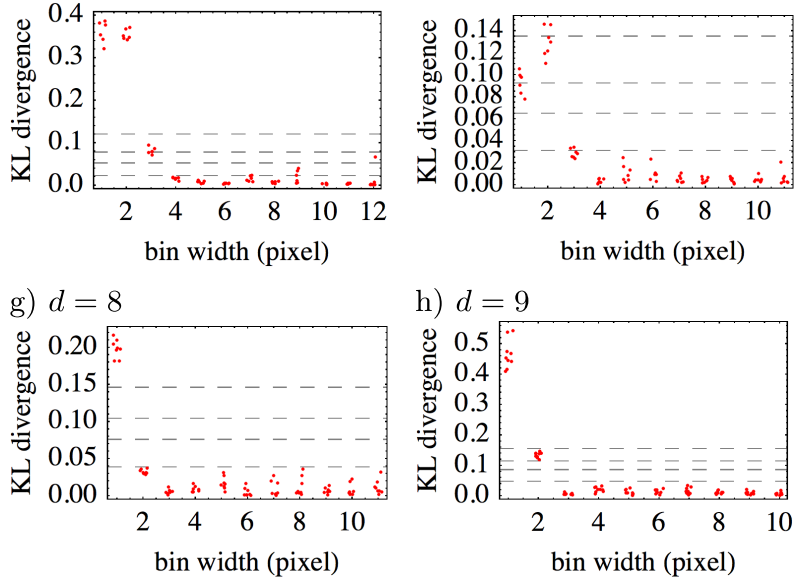

h) $d=9$
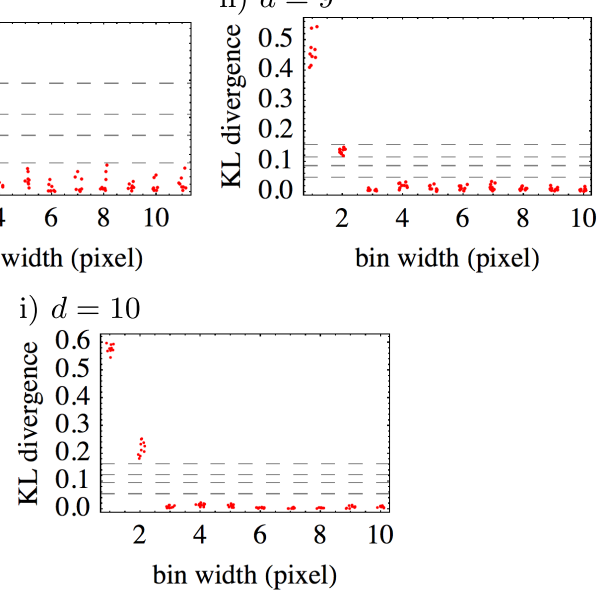

Figure 7: The Kullback-Leibler divergences of the distributions obtained by preparing an eigenstate of $\hat{\Omega}_{k}^{0}$ and performing the measurements described by $\left\{\hat{\Omega}_{k^{\prime}}^{23^{\circ}}\right\}$. Each figure summarizes the results for one dimension. The horizontal axes are the bin widths in terms of number of pixels. The vertical axes are the KL divergences from the uniform distributions. The top dashed line in each figure identifies the KL divergence value above which lie $80 \%$ of the probability distributions simulated in the previous section. Analogously, the following lines from top to bottom determine the limits for $90 \%$, $95 \%$ and $99 \%$ of values respectively (note that in the first two figures, only the lines corresponding to the $80 \%$ and $90 \%$ limits are seen). The error bars have been omitted for being about the size of the points. 
divergences from the uniform distribution obtained for these measurements, one for each $d$. On the horizontal axes are the bin widths, in pixels, used for the preparation masks. The bin width of the measurement masks can be obtained from Eq. (10). We can see that for the smallest bin widths, the data tend to not be as reliable. Nevertheless, for larger bin widths the KL divergences are clearly near zero.

Again, we can see that, even for a relatively small angle in phase-space between the variables, we obtain probability distributions very close to the uniform distribution, showing the unbiasedness of the measurements.

\section{DISCUSSION}

Periodic coarse graining can be used to recover mutual unbiasedness, a property that is not present in physical realization of the standard coarse-grained versions of phase-space observables. We have shown that, for a periodic coarse graining given by mask functions defined in Eq. (4), and in the particular case of $m_{1}=m_{2}=m_{3}$ [see Eq. (12)], up to three mutually unbiased bases can be defined. The open question remains as to whether one can define more mutually unbiased measurements for other cases, and is an interesting topic to be investigated.

An optics experiment was performed to demonstrate these results. The optical fractional Fourier transform was used to prepare and measure periodic coarse-grained versions of a symmetric phase space triple of operators. Very good agreement was found between theory and experiment for all measurement dimensionality tested in our experiment (from 2 to 10). In addition, we experimentally tested mutual unbiasedness for periodic coarse graining of measurements that correspond to phase-space variables that are separated by only a $23^{\circ}$ rotation in phase space.

Our theoretical and experimental results contribute to the further understanding of the relation between continuous and discrete quantum mechanics, and could prove useful in the discover of new uncertainty relations, and in adapting quantum information protocols to continuous variable systems.

\section{Acknowledgments}

The authors acknowledge financial support from the Brazilian funding agencies CNPq, CAPES and FAPERJ, and the National Institute of Science and Technology Quantum Information. Ł.R. acknowledges financial support by grant number 2014/13/D/ST2/01886 of the National Science Center, Poland.

Appendix A: Derivation of Eq. 6

We start the derivation by employing the Fourier series expansion (5) of the periodic mask function

$$
\hat{\Omega}_{k}^{\theta}=\sum_{N \in \mathbb{Z}} f_{N} e^{i N \varphi_{k}} \int d q_{\theta} e^{i \frac{N \tau_{\theta} q_{\theta}}{\sin \Delta \theta}}\left|q_{\theta}\right\rangle\left\langle q_{\theta}\right|
$$

with $\varphi_{k}=-2 \pi k / d-q_{\theta}^{\mathrm{cen}} \tau_{\theta} / \sin \Delta \theta$. Using the completeness relation $1=\int d q_{\theta^{\prime}}\left|q_{\theta^{\prime}}\right\rangle\left\langle q_{\theta^{\prime}}\right|$, we can change the basis from $\left|q_{\theta}\right\rangle$ to its rotated counterpart $\left|q_{\theta^{\prime}}\right\rangle$

$$
\hat{\Omega}_{k}^{\theta}=\sum_{N \in \mathbb{Z}} f_{N} e^{i N \varphi_{k}} \int d q_{\theta^{\prime}} \int d \tilde{q}_{\theta^{\prime}} Q\left(q_{\theta^{\prime}}, \tilde{q}_{\theta^{\prime}}\right)\left|q_{\theta^{\prime}}\right\rangle\left\langle\tilde{q}_{\theta^{\prime}}\right|
$$

where

$$
Q\left(q_{\theta^{\prime}}, \tilde{q}_{\theta^{\prime}}\right)=\int d q_{\theta} e^{i \frac{N \tau_{\theta} q_{\theta}}{\sin \Delta \theta}} \mathcal{F}\left(q_{\theta^{\prime}}, q_{\theta}\right) \mathcal{F}\left(q_{\theta}, \tilde{q}_{\theta^{\prime}}\right)
$$

The variable $\tilde{q}_{\theta^{\prime}}$ has the same physical meaning as $q_{\theta^{\prime}}$ and the tilde on top of it has only been introduced to distinguish both integration variables.

An explicit form of the above kernel is

$$
Q\left(q_{\theta^{\prime}}, \tilde{q}_{\theta^{\prime}}\right)=\frac{e^{i \frac{\cot \Delta \theta}{2}\left(q_{\theta^{\prime}}^{2}-\tilde{q}_{\theta^{\prime}}^{2}\right)}}{2 \pi|\sin \Delta \theta|} \int d q_{\theta} e^{i \frac{q_{\theta}}{\sin \Delta \theta}\left(N \tau_{\theta}+\tilde{q}_{\theta^{\prime}}-q_{\theta^{\prime}}\right)}
$$

One can immediately perform the integration with respect to $q_{\theta}$ as it leads to the Dirac delta function

$$
Q\left(q_{\theta^{\prime}}, \tilde{q}_{\theta^{\prime}}\right)=e^{i \frac{\cot \Delta \theta}{2}\left(q_{\theta^{\prime}}^{2}-\tilde{q}_{\theta^{\prime}}^{2}\right)} \delta\left(N \tau_{\theta}+\tilde{q}_{\theta^{\prime}}-q_{\theta^{\prime}}\right)
$$

In the last step, one needs to plug the above expression into Eq. A2, perform the integration with respect to $\tilde{q}_{\theta^{\prime}}$ and simplify accordingly.
[1] J. Schwinger. Unitary operator bases. Proc. Natl. Acad. Sci., 46:570, 1960.

[2] A. Kalev and G. Gour. Mutually unbiased measurements in finite dimensions. New J. Phys., 16(5):053038, 2014.

[3] K. Kraus. Complementary observables and uncertainty relations. Phys. Rev. D, 35:3070, 1987.
[4] N. Gisin, G. Ribordy, W. Tittel, and H. Zbinden. Quantum cryptography. Rev. Mod. Phys., 74:145, 2002.

[5] P. J. Coles, M. Berta, M. Tomamichel, and S. Wehner. Entropic uncertainty relations and their applications. Rev. Mod. Phys., 89:015002, 2017.

[6] A. Fernández-Pérez, A. B. Klimov, and C. Saavedra. 
Quantum process reconstruction based on mutually unbiased basis. Phys. Rev. A, 83:052332, 2011.

[7] D. Giovannini, J. Romero, J. Leach, A. Dudley, A. Forbes, and M. J. Padgett. Characterization of highdimensional entangled systems via mutually unbiased measurements. Phys. Rev. Lett., 110:143601, 2013.

[8] C. Spengler, M. Huber, S. Brierley, T. Adaktylos, and B. C. Hiesmayr. Entanglement detection via mutually unbiased bases. Phys. Rev. A, 86:022311, 2012.

[9] E. C. Paul, D. S. Tasca, Ł. Rudnicki, and S. P. Walborn. Detecting entanglement of continuous variables with three mutually unbiased bases. Phys. Rev. A, 94:012303, 2016.

[10] D. Sauerwein, C. Macchiavello, L. Maccone, and B. Kraus. Multipartite correlations in mutually unbiased bases. Phys. Rev. A, 95:042315, 2017.

[11] E. G. Cavalcanti, S. J. Jones, H. M. Wiseman, and M. D. Reid. Experimental criteria for steering and the einsteinpodolsky-rosen paradox. Phys. Rev. A, 80:032112, 2009.

[12] S. P. Walborn, A. Salles, R. M. Gomes, F. Toscano, and P. H. Souto Ribeiro. Revealing hidden einstein-podolskyrosen nonlocality. Phys. Rev. Lett., 106:130402, 2011.

[13] D. S. Tasca, E. Rudnicki, R. M. Gomes, F. Toscano, and S. P. Walborn. Reliable entanglement detection under coarse-grained measurements. Phys. Rev. Lett., 110:210502, 2013.

[14] J. Schneeloch, P. B. Dixon, G. A. Howland, C. J. Broadbent, and J. C. Howell. Violation of continuous-variable einstein-podolsky-rosen steering with discrete measurements. Phys. Rev. Lett., 110:130407, 2013.

[15] J. Schneeloch, C. J. Broadbent, S. P. Walborn, E. G. Cavalcanti, and J. C. Howell. Einstein-podolsky-rosen steering inequalities from entropic uncertainty relations. Phys. Rev. A, 87:062103, 2013.

[16] P. Skrzypczyk, M. Navascués, and D. Cavalcanti. Quantifying einstein-podolsky-rosen steering. Phys. Rev. Lett., 112:180404, 2014.

[17] M. Marciniak, A. Rutkowski, Z. Yin, M. Horodecki, and R. Horodecki. Unbounded violation of quantum steering inequalities. Phys. Rev. Lett., 115:170401, 2015.

[18] H. Zhu, M. Hayashi, and L. Chen. Universal steering criteria. Phys. Rev. Lett., 116:070403, 2016.

[19] G. Vallone, D. G. Marangon, M. Tomasin, and P. Villoresi. Quantum randomness certified by the uncertainty principle. Phys. Rev. A, 90:052327, 2014.

[20] T. Durt, B.-G. Englert, I. Bengtsson, and K. Życzkowski. On mutually unbiased bases. Int. J. Quant. Inf., 08(04):535, 2010.

[21] S. Weigert and M. Wilkinson. Mutually unbiased bases for continuous variables. Phys. Rev. A, 78:020303, 2008.

[22] S. L. Braunstein and P. van Loock. Quantum information with continuous variables. Rev. Mod. Phys., 77:513, 2005.
[23] Ł. Rudnicki, S. P. Walborn, and F. Toscano. Heisenberg uncertainty relation for coarse-grained observables. Europhys. Lett., 97(3):38003, 2012.

[24] Ł. Rudnicki, S. P. Walborn, and F. Toscano. Optimal uncertainty relations for extremely coarse-grained measurements. Phys. Rev. A, 85:042115, 2012.

[25] D. S. Tasca, P. Sánchez, S. P. Walborn, and Ł. Rudnicki. Mutual unbiasedness in coarse-grained continuous variables. Phys. Rev. Lett., 120:040403, 2018.

[26] Y. Aharonov, H. Pendleton, and A. Petersen. Modular variables in quantum theory. Int. J. Theor. Phys., 2:213, 1969.

[27] P. Busch and P. J. Lahti. To what extent do position and momentum commute? Phys. Lett. A, 115:259, 1986.

[28] H. Reiter and W. Thirring. Are $\mathrm{x}$ and $\mathrm{p}$ incompatible observables? Found. Phys., 19(8):1037, 1989.

[29] C. Gneiting and K. Hornberger. Detecting entanglement in spatial interference. Phys. Rev. Lett., 106:210501, 2011.

[30] M. A. D. Carvalho, J. Ferraz, G. F. Borges, P.-L. de Assis, S. Pádua, and S. P. Walborn. Experimental observation of quantum correlations in modular variables. Phys. Rev. A, 86:032332, 2012.

[31] P. Vernaz-Gris, A. Ketterer, A. Keller, S. P. Walborn, T. Coudreau, and P. Milman. Continuous discretization of infinite-dimensional hilbert spaces. Phys. Rev. A, 89:052311, 2014.

[32] A. Ketterer, A. Keller, S. P. Walborn, T. Coudreau, and P. Milman. Quantum information processing in phase space: A modular variables approach. Phys. Rev. A, 94:022325, 2016.

[33] A. Laversanne-Finot, A. Ketterer, M. R. Barros, S. P. Walborn, T. Coudreau, A. Keller, and P. Milman. General conditions for maximal violation of noncontextuality in discrete and continuous variables. $J$. Phys. A, 50(15):155304, 2015.

[34] H. M. Ozaktas, Z. Zalevsky, and M. A. Kutay. The Fractional Fourier Transform: with Applications in Optics and Signal Processing. John Wiley and Sons Ltd, New York, 2001.

[35] V. V. Dodonov. Variance uncertainty relations without covariances for three and four observables. Phys. Rev. A, 97:022105, 2018.

[36] A. W. Lohmann. Image rotation, wigner rotation, and the fractional fourier transform. J. Opt. Soc. Am. A, 10:2181, 1993.

[37] D. S. Tasca, R. M. Gomes, F. Toscano, P. H. Souto Ribeiro, and S. P. Walborn. Continuous-variable quantum computation with spatial degrees of freedom of photons. Phys. Rev. A, 83:052325, 2011.

[38] We assume here that 0 does not belong to $\mathbb{N}$. 